\title{
BINARY BELL POLYNOMIALS AND DARBOUX COVARIANT LAX PAIRS
}

\author{
F. LAMBERT \\ Theoretical Physics Division, Vrije Universiteit Brussel, Pleinlaan 2, B-1050 Brussels, Belgium \\ S. LEBLE \\ Theoretical Physics Dept, Kaliningrad State University, Russia and \\ Theoretical and Mathematical Physics Dept, Technical University of Gdansk, Poland \\ and J. SPRINGAEL \\ Theoretical Physics Division, Vrije Universiteit Brussel, Pleinlaan 2, B-1050 Brussels, Belgium
}

(Received 14 March, 2000; revised 12 September, 2000)

1991 Mathematics Subject Classification. 37K10, 37K15, 37K40.

1. Introduction. Hirota representations of soliton equations have proved very useful. They produced many of the known families of multisoliton solutions, and have often led to a disclosure of the underlying Lax systems and infinite sets of conserved quantities $[\mathbf{1}, \mathbf{2}]$.

A striking feature is the ease with which direct insight can be gained into the nature of the eigenvalue problem associated with soliton equations derivable from a quadratic Hirota equation (for a single Hirota function), such as the KdV equation or the Boussinesq equation. A key element is the bilinear Bäcklund transformation (BT) which can be obtained straight away from the Hirota representation of these equations, through decoupling of a related "two field condition" by means of an appropriate constraint of minimal weight. Details of this procedure have been reported elsewhere $[\mathbf{3 , 4}]$. The main point is that bilinear BT's are obtained systematically, without the need of tricky "exchange formulas" [1]. They arise in the form of "Y-systems", each equation of which belongs to a linear space spanned by a basis of binary Bell polynomials ( $\mathcal{Y}$-polynomials) [5].

An important element is the logarithmic linearizability of $\mathcal{Y}$-systems, which implies that each bilinear BT can be mapped onto a corresponding linear system of Lax type. However, it turns out that these linear systems involve differential operators which, even in the simplest case, do not constitute a Darboux covariant Lax pair. See Chapters 2 and 3 of [6]. This fact prevents us from obtaining large classes of solutions by direct application of the powerful Darboux machinery to the systems which arise by straightforward linearization of the $\mathcal{Y}$-systems.

Here we present a simple scheme by means of which this difficulty can be resolved for a variety of soliton equations which admit a bilinear BT that comprises a constraint of lowest possible weight (weight 2). Darboux covariant Lax pairs for the $\mathrm{KdV}$, Boussinesq and $\mathrm{KdV}_{5}$ equations are obtained in a unified manner, by exploiting the relations between the coefficients of linear differential operators connected by a classical Darboux transformation. We show, in particular, that the concept of Darboux covariance may be regarded as an elementary tool capable of 
generating families of $1+1$ dimensional soliton systems, exhibiting their close connection within a higher dimensional hierarchy (KP).

We start our discussion by recalling the main properties of the $\mathcal{Y}$-polynomials (derived in [5]) and by indicating how the use of the $\mathcal{Y}$-basis can lead systematically from the original nonlinear partial differential equations to the associated linear systems. The example of the Lax equation is instructive since this fifth order equation cannot be derived from a single quadratic Hirota equation.

2. Binary Bell polynomials. The binary polynomials that we use are defined in terms of the exponential Bell polynomials [7]

$$
Y_{m x, n t}(f) \equiv e^{-f} \partial_{x}^{m} \partial_{t}^{n} e^{f}
$$

as follows:

$$
\left.\mathcal{Y}_{m x, n t}(v, w) \equiv Y_{m x, n t}(f)\right|_{f_{p x, q t}=\left\{\begin{array}{l}
v_{p x, q t} \text { if } p+q \text { is odd, } \\
w_{p x, q t} \text { if } p+q \text { is even, }
\end{array}\right.}
$$

with the understanding that $f_{p x, q t} \equiv \partial_{x}^{p} \partial_{t}^{q} f$.

They inherit the easily recognizable partitional structure of the Bell polynomials

$$
\begin{aligned}
& \mathcal{Y}_{x}(v)=v_{x}, \quad \mathcal{Y}_{2 x}(v, w)=w_{2 x}+v_{x}^{2}, \quad \mathcal{Y}_{x, t}(v, w)=w_{x t}+v_{x} v_{t}, \\
& \mathcal{Y}_{3 x}(v, w)=v_{3 x}+3 v_{x} w_{2 x}+v_{x}^{3}, \cdots
\end{aligned}
$$

The link between $\mathcal{Y}$-polynomials and the standard Hirota expressions

$$
D_{x}^{p} D_{t}^{q} G^{\prime} \cdot G \equiv\left(\partial_{x}-\partial_{x^{\prime}}\right)^{p}\left(\partial_{t}-\partial_{t^{\prime}}\right)^{q} G^{\prime}(x, t) G\left(x^{\prime}, t^{\prime}\right)_{\left.\right|_{x^{\prime}=x, t^{\prime}=t}}
$$

is given by the identity

$$
\mathcal{Y}_{m x, n t}\left(v=\ln G^{\prime} / G, w=\ln G^{\prime} G\right) \equiv\left(G^{\prime} G\right)^{-1} D_{x}^{m} D_{t}^{n} G^{\prime} \cdot G .
$$

In the particular case $G^{\prime}=G$ one has

$$
G^{-2} D_{x}^{m} D_{t}^{n} G \cdot G \equiv \mathcal{Y}_{m x, n t}(0, Q=2 \ln G) \equiv\left\{\begin{array}{l}
0 \text { if } m+n \text { is odd } \\
P_{m x, n t}(Q) \text { if } m+n \text { is even }
\end{array}\right.
$$

the $P$-polynomials being characterized by an equally recognizable "even part" partitional structure

$$
\begin{aligned}
& P_{2 x}(Q)=Q_{2 x}, \quad P_{x, t}(Q)=Q_{x t}, \quad P_{4 x}(Q)=Q_{4 x}+3 Q_{2 x}^{2}, \\
& P_{6 x}(Q)=Q_{6 x}+15 Q_{2 x} Q_{4 x}+15 Q_{2 x}^{3}, \cdots
\end{aligned}
$$

A crucial property of the $\mathcal{Y}$-polynomials is related to the transformation $w=v+Q, v=\ln \psi:$ 


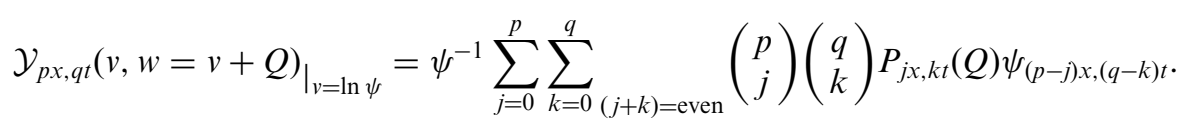

It should also be noticed that polynomials $\mathcal{Y}_{p x, q t}(v, w)$, constructed with the derivatives of dimensionless variables $v$ and $w$, are homogeneous expressions of weight $p+q r$ if $r$ stands for the dimension of $t$ (the dimension of $x$ is chosen equal to 1 ).

3. $\mathcal{Y}$-systems associated with sech squared soliton equations. We consider three examples of sech squared soliton equations with order ranging from 3 to 5 : $\mathrm{KdV}$, Boussinesq, and $\mathrm{KdV}_{5}$.

The simplest one is the $\mathrm{KdV}$ equation

$$
\operatorname{KdV}(u) \equiv u_{t}+u_{3 x}+6 u u_{x}=0 .
$$

Its invariance under the scale transformation

$$
x \rightarrow \lambda x, \quad t \rightarrow \lambda^{3} t, \quad u \rightarrow \lambda^{-2} u
$$

shows that $u$ has the dimension -2. A dimensionless field $Q$ can be introduced by setting $u=c Q_{2 x}$, with $c$ a dimensionless parameter to be determined. The resulting equation for $Q$ can be derived from the "potential" equation

$$
Q_{x t}+Q_{4 x}+3 c Q_{2 x}^{2}=0
$$

which can be cast into the form

$$
E(Q) \equiv P_{x t}(Q)+\left.P_{4 x}(Q) \equiv G^{-2}\left(D_{x} D_{t}+D_{x}^{4}\right) G \cdot G\right|_{G=\exp (Q / 2)}=0
$$

by setting $c=1$.

The well known Hirota "two field condition" on $G$ and $G^{\prime}$, to be satisfied as a differential consequence of a bilinear BT (that one must find) takes the form [1]

$$
G^{\prime-2}\left(D_{x} D_{t}+D_{x}^{4}\right) G^{\prime} \cdot G^{\prime}-G^{-2}\left(D_{x} D_{t}+D_{x}^{4}\right) G \cdot G=0 .
$$

It corresponds to the following condition on $Q=2 \ln G=w-v$ and $Q^{\prime}=2 \ln G^{\prime}=w+v$ :

$$
\begin{aligned}
& E(w+v)-E(w-v)=2\left(v_{x t}+v_{4 x}+6 v_{2 x} w_{2 x}\right) \\
& \equiv 2\left\{\partial_{x}\left[\mathcal{Y}_{t}(v)+\mathcal{Y}_{3 x}(v, w)\right]+6 \mathrm{~W}\left[\mathcal{Y}_{2 x}(v, w), \mathcal{Y}_{x}(v)\right]\right\}=0 .
\end{aligned}
$$

This condition can easily be decoupled into a pair of equations in the form of linear combinations of $\mathcal{Y}$-polynomials set equal to zero. It suffices to impose such a constraint on $v$ and $w\left(p_{j}, q_{j}=\right.$ integer or zero, $c_{j}=$ constant $)$

$$
\sum_{j} c_{j} \mathcal{Y}_{p_{j} x, q_{j} t}(v, w)=0
$$


of lowest possible order (or weight).

The simplest possible choice is a constraint of weight 2

$$
\mathcal{Y}_{2 x}(v, w) \equiv w_{2 x}+v_{x}^{2}=0
$$

In order to obtain a parameter dependent decomposition we rather impose the condition

$$
\mathcal{Y}_{2 x}(v, w)=\lambda, \quad \lambda=\text { arbitrary parameter of weight } 2 .
$$

It leads us to the following $\mathcal{Y}$-system

$$
\begin{aligned}
& \mathcal{Y}_{2 x}(v, w)-\lambda=0, \\
& \mathcal{Y}_{t}(v)+\mathcal{Y}_{3 x}(v, w)+3 \lambda \mathcal{Y}_{x}(v)=0,
\end{aligned}
$$

the compatibility of which is subject to that of the corresponding system for $\psi$ (setting $w=v+Q, v=\ln \psi$ and using relation (9))

$$
\begin{aligned}
& \left(\mathcal{L}_{2}-\lambda\right) \psi \equiv \psi_{2 x}+\left(Q_{2 x}-\lambda\right) \psi=0, \\
& \left(\partial_{t}+\mathcal{L}_{3}\right) \psi \equiv \psi_{t}+\psi_{3 x}+3\left(Q_{2 x}+\lambda\right) \psi_{x}=0,
\end{aligned}
$$

i.e. to the ( $\lambda$-independent) condition

$$
\left(Q_{x t}+Q_{4 x}+3 Q_{2 x}\right)_{x} \equiv \partial_{x} E(Q)=0 .
$$

The bilinear equivalent of the $\mathcal{Y}$-system (19) is obtained by means of relation (6)

$$
\begin{aligned}
& D_{x}^{2} G^{\prime} \cdot G=\lambda G^{\prime} G, \\
& \left(D_{t}+D_{x}^{3}+3 \lambda D_{x}\right) G^{\prime} \cdot G=0 .
\end{aligned}
$$

It is the bilinear BT for $\mathrm{KdV}$ proposed by Hirota [1].

A similar analysis can be applied to the Boussinesq equation

$$
\mathrm{Bq}(u) \equiv u_{2 t}-u_{4 x}+3\left(u^{2}\right)_{2 x}=0 .
$$

It shows that this equation can be derived from a potential version, obtained by setting $u=-Q_{2 x}$, which can be cast into the form

$$
E(Q) \equiv P_{2 t}(Q)-P_{4 x}(Q) \equiv G^{-2}\left(D_{t}^{2}-D_{x}^{4}\right) G \cdot G_{\left.\right|_{G=\exp (Q / 2)}}=0 .
$$

The corresponding two field condition

$$
\begin{aligned}
E\left(Q^{\prime}=w+v\right)-E(Q=w+v) & \equiv 2\left(v_{2 t}-v_{4 x}-6 v_{2 x} w_{2 x}\right) \\
& =-2 \partial_{x} \mathcal{Y}_{3 x}(v, w)+2 v_{2 t}+6 W\left[\mathcal{Y}_{2 x}(v, w), \mathcal{Y}_{x}(v)\right]=0
\end{aligned}
$$

can still be decoupled into a pair of equations of the form (16) by means of a $\mathcal{Y}$ constraint of weight 2 (notice that in this case the dimension of $t=2$, so that we dispose of two $\mathcal{Y}$-polynomials of weight 2) 
$\mathcal{Y}_{t}(v)+a \mathcal{Y}_{2 x}(v, w)=0, \quad a=$ dimensionless constant to be determined.

The decoupling requires $a^{2}=-3$ and produces the following parameter dependent $\mathcal{Y}$-system $(\lambda=$ integration constant $)$ :

$$
\begin{aligned}
& \mathcal{Y}_{t}+a \mathcal{Y}_{2 x}(v, w)=0, \\
& a \mathcal{Y}_{x, t}(v, w)+\mathcal{Y}_{3 x}(v, w)=\lambda . \quad\left(a^{2}=-3\right)
\end{aligned}
$$

The corresponding bilinear system

$$
\begin{aligned}
& \left(D_{t}+a D_{x}^{2}\right) G^{\prime} \cdot G=0 \\
& \left(a D_{x} D_{t}+D_{x}^{3}-\lambda\right) G^{\prime} \cdot G=0
\end{aligned}
$$

is the bilinear BT for Boussinesq obtained by Nimmo and Freeman [8]. Its compatibility is subject to that of the linear equivalent to the system (26):

$$
\begin{aligned}
& \psi_{t}+a \psi_{2 x}+a Q_{2 x} \psi=0, \\
& a \psi_{x t}+\psi_{3 x}+3 Q_{2 x} \psi_{x}+\left(a Q_{x t}-\lambda\right) \psi=0, \quad\left(a^{2}=-3\right),
\end{aligned}
$$

i.e. to the following potential version of the Boussinesq equation:

$$
\operatorname{PBq}(Q) \equiv\left(Q_{2 t}-Q_{4 x}-3 Q_{2 x}^{2}\right)_{x}=0
$$

We now consider the $\mathrm{KdV}_{5}$ equation

$$
\mathrm{KdV}_{5}(u) \equiv u_{t}+u_{5 x}+10 u u_{3 x}+20 u_{x} u_{2 x}+30 u^{2} u_{x}=0
$$

Setting $u=c Q_{2 x}$ it is found that it can be derived from the potential equation:

$$
E_{c}(Q) \equiv Q_{x t}+Q_{6 x}+10 c Q_{2 x} Q_{4 x}+5 c Q_{3 x}^{2}+10 c^{2} Q_{2 x}^{3}=0 .
$$

The left hand side of this equation is homogeneous of weight 6 , but there is no value of $c$ such that it be expressible as a linear combination of the weight 6 polynomials $P_{6 x}(Q)$ and $P_{x t}(Q)$.

Setting $c=1$, we may nevertheless consider the two field condition

$$
E_{1}(w+v)-E_{1}(w-v) \equiv 2\left\{\partial_{x}\left[\mathcal{Y}_{t}(v)+\mathcal{Y}_{5 x}(v, w)\right]+R(v, w)\right\}=0,
$$

with

$$
\begin{aligned}
R(v, w)= & -5\left(v_{x} w_{5 x}-v_{2 x} w_{4 x}+6 v_{x} w_{2 x} w_{3 x}+2 v_{x}^{3} w_{3 x}-3 v_{2 x} w_{2 x}^{2}\right. \\
& \left.+6 v_{x}^{2} v_{2 x} w_{2 x}+4 v_{x} v_{2 x} v_{3 x}+2 v_{x}^{2} v_{4 x}+v_{x}^{4} v_{2 x}-2 v_{2 x}^{3}\right) .
\end{aligned}
$$

Eliminating $w_{2 x}$ (and its derivatives) by means of the weight 2 constraint (18) it is found that the condition (32) can be decoupled into the following $\mathcal{Y}$-system:

$$
\begin{aligned}
& \mathcal{Y}_{2 x}(v, w)=\lambda, \\
& \mathcal{Y}(v)+\mathcal{Y}_{5 x}(v, w)+15 \lambda^{2} \mathcal{Y}_{x}(v)=0
\end{aligned}
$$


Its compatibility is subject to that of the corresponding linear system:

$$
\begin{aligned}
& \psi_{2 x}+\left(Q_{2 x}-\lambda\right) \psi=0, \\
& \psi_{t}+\mathcal{L}_{5} \psi=0, \quad \mathcal{L}_{5}=\partial_{x}^{5}+10 Q_{2 x} \partial_{x}^{3}+5\left(Q_{4 x}+3 Q_{2 x}^{2}+3 \lambda^{2}\right) \partial_{x},
\end{aligned}
$$

i.e. to the condition

$$
\left(Q_{x t}+Q_{6 x}+10 Q_{2 x} Q_{4 x}+5 Q_{3 x}^{2}+10 Q_{2 x}^{3}\right)_{x} \equiv \partial_{x} E_{1}(Q)=0 .
$$

4. Darboux covariant Lax pairs. Let us now go back to the KdV equation (10) and the associated linear system (20). It comprises the second order eigenvalue equation with the well known Darboux property [6], according to which (non vanishing) solutions $\phi$ of this equation produce gauge transformations [9]

$$
G_{\phi}=\phi \partial_{x} \phi^{-1}=\partial_{x}-\sigma, \quad \sigma=\partial_{x} \ln \phi,
$$

which map $\mathcal{L}_{2}(Q)=\partial_{x}^{2}+Q_{2 x}$ onto a similar operator:

$$
\widetilde{\mathcal{L}}_{2} \equiv G_{\phi} \mathcal{L}_{2}(Q) G_{\phi}^{-1} \equiv \mathcal{L}_{2}(\widetilde{Q}) \quad \text { with } \quad \widetilde{Q}=Q+2 \ln \phi
$$

A similar property does not hold for the evolution equation in (20). However, it is easy to see that transformations $G_{\phi}$ generated by solutions $\phi$ of the third order evolution equation

$$
\left(\partial_{t}+L_{3}\right) \phi=0, \quad L_{3}=\partial_{x}^{3}+b_{2} \partial_{x}+b_{3},
$$

map the operator $\partial_{t}+L_{3}$ onto the similar

$$
G_{\phi}\left(\partial_{t}+L_{3}\right) G_{\phi}^{-1}=\partial_{t}+\widetilde{L}_{3}, \quad \widetilde{L}_{3}=\partial_{x}^{3}+\tilde{b}_{2} \partial_{x}+\tilde{b}_{3},
$$

with

$$
\Delta b_{2} \equiv \widetilde{b}_{2}-b_{2}=3 \sigma_{x} \quad \text { and } \quad \Delta b_{3} \equiv \widetilde{b}_{3}-b_{3}=b_{2, x}+\sigma \Delta b_{2}+3 \sigma_{2 x}
$$

This follows from equation (39) and more particularly from its differential consequence

$$
\sigma_{t}+\partial_{x}\left[\sigma_{2 x}+3 \sigma \sigma_{x}+\sigma^{3}+b_{2} \sigma+b_{3}\right]=0 .
$$

The next step is to look for a system, equivalent to the system (20), in which the eigenvalue equation

$$
\mathcal{L}_{2}(Q) \phi=\lambda \phi
$$

is accompanied by a Darboux covariant third order evolution equation (39). The problem is to find a third order operator $L_{3}$, with appropriate coefficients $b_{2}(Q)$ and $b_{3}(Q)$, such that $\partial_{t}+L_{3}(Q)$ be mapped, by gauge transformations (37) generated by (non vanishing) solutions $\phi$ of the system $(43,39)$, onto a similar operator, $\partial_{t}+\widetilde{L}_{3}(Q)$, which satisfies the covariance condition 


$$
\widetilde{L}_{3}(Q)=L_{3}(\widetilde{Q}=Q+\Delta Q), \quad \Delta Q=2 \ln \phi .
$$

It suffices to determine $b_{2}$ and $b_{3}$ in the form of polynomial expressions in terms of derivatives of $Q$ (of order $r>1$ )

$$
b_{i}=F_{i}\left(Q_{2 x}, Q_{3 x}, \cdots\right), \quad i=2,3,
$$

such that

$$
\Delta F_{i} \equiv F_{i}\left(Q_{2 x}+\Delta Q_{2 x}, Q_{3 x}+\Delta Q_{3 x}, \cdots\right)-F_{i}\left(Q_{2 x}, Q_{3 x}, \cdots\right)=\Delta b_{i},
$$

with $\Delta Q_{r x}=2(\ln \phi)_{r x}$, the $\Delta b_{i}$ being determined by the relations (41).

Thus, in order to satisfy the first condition:

$$
\Delta F_{2}=F_{2, Q_{2 x}} \cdot \Delta Q_{2 x}+\cdots=3 \sigma_{x}=\frac{3}{2} \Delta Q_{2 x},
$$

one chooses

$$
b_{2}=F_{2}\left(Q_{2 x}\right) \equiv \frac{3}{2} Q_{2 x}+c_{2}, \quad c_{2}=\text { arbitrary constant }
$$

The expression (41) for $\Delta b_{3}$ then becomes

$$
\Delta b_{3}=\frac{3}{2} Q_{3 x}+3 \sigma \sigma_{x}+3 \sigma_{2 x},
$$

from which $Q_{3 x}$ can be eliminated on account of equation (43)

$$
Q_{3 x}=-\sigma_{2 x}-2 \sigma \sigma_{x} .
$$

In view of the resulting expression

$$
\Delta b_{3}=\frac{3}{2} \sigma_{2 x}=\frac{3}{4} \Delta Q_{3 x}
$$

it is clear that the second condition

$$
\Delta F_{3}=F_{3, Q_{2 x}} \cdot \Delta Q_{2 x}+F_{3, Q_{3 x}} \cdot \Delta Q_{3 x}+\cdots=\frac{3}{4} \Delta Q_{3 x}
$$

is satisfied if one chooses

$$
b_{3}=F_{3}\left(Q_{3 x}\right) \equiv \frac{3}{4} Q_{3 x}+c_{3}, \quad c_{3}=\text { arbitrary constant }
$$

Setting $c_{2}=c_{3}=0$ for simplicity, we find the following Darboux covariant evolution equation

$$
\left[\partial_{t}+L_{3, \mathrm{cov}}(Q)\right] \phi=0, \quad L_{3, \mathrm{cov}}(Q)=\partial_{x}^{3}+\frac{3}{2} Q_{2 x} \partial_{x}+\frac{3}{4} Q_{3 x} .
$$


The system $(43,54)$ is a Darboux covariant equivalent to the former system $(20)$ as

$$
L_{3, \operatorname{cov}}(Q)=\frac{1}{4}\left[\mathcal{L}_{3}+3 \partial_{x}\left(\mathcal{L}_{2}-\lambda\right)\right]
$$

The operator $L_{3 \text {,cov }}(Q)$ coincides precisely to the third order operator which gives rise to the $\mathrm{KdV}$ equation in the Lax formalism [10]

$$
\left[\partial_{t}+L_{3, \operatorname{cov}}(Q), \mathcal{L}_{2}(Q)\right]=-\frac{1}{4}\left(Q_{x t}+Q_{4 x}+3 Q_{2 x}^{2}\right)_{x} .
$$

The analogy between the Lax formalism and the requirement of Darboux covariance can be further disclosed by considering a Darboux covariant equivalent to the system (35), i.e. by looking for a system in which the eigenvalue equation (43) is accompanied by a Darboux covariant fifth order evolution equation $\left(c_{1}=\right.$ constant)

$$
\left[\partial_{t}+L_{5}(Q)\right] \phi=0, \quad L_{5}(Q)=\partial_{x}^{5}+c_{1} \partial_{x}^{4}+b_{2}(Q) \partial_{x}^{3}+b_{3}(Q) \partial_{x}^{2}+b_{4}(Q) \partial_{x}+b_{5}(Q) .
$$

A transformation $G_{\phi}$ generated by a (non vanishing) solution $\phi$ of the system $(43,57)$ maps $\partial_{t}+L_{5}(Q)$ onto the similar $\partial_{t}+\widetilde{L}_{5}(Q)$, where

$$
\widetilde{L}_{5}(Q)=\partial_{x}^{5}+c_{1} \partial_{x}^{4}+\tilde{b}_{2}(Q) \partial_{x}^{3}+\tilde{b}_{3}(Q) \partial_{x}^{2}+\tilde{b}_{4}(Q) \partial_{x}+\tilde{b}_{5}(Q)
$$

with

$$
\begin{aligned}
\Delta b_{2} & \equiv \tilde{b}_{2}-b_{2}=5 \sigma_{x}=\frac{5}{2} \Delta Q_{2 x}, \\
\Delta b_{3} & \equiv \widetilde{b}_{3}-b_{3}=b_{2, x}+\sigma \Delta b_{2}+4 c_{1} \sigma_{x}+10 \sigma_{2 x}, \\
\Delta b_{4} & \equiv \widetilde{b}_{4}-b_{4}=b_{3, x}+\sigma \Delta b_{3}+3 \sigma_{x} \widetilde{b}_{2}+6 c_{1} \sigma_{2 x}+10 \sigma_{3 x}, \\
\Delta b_{5} & \equiv \widetilde{b}_{5}-b_{5}=b_{4, x}+\sigma \Delta b_{4}+2 \sigma_{x} \widetilde{b}_{3}+3 \sigma_{2 x} \widetilde{b}_{2}+4 c_{1} \sigma_{3 x}+5 \sigma_{4 x} .
\end{aligned}
$$

These relations can again be used in a straightforward manner to determine polynomial expressions $b_{i}(Q), i=2,3,4,5$, which satisfy the covariance requirement

$$
\widetilde{b}_{i}(Q) \equiv b_{i}(Q)+\Delta b_{i}=b_{i}(\widetilde{Q}=Q+2 \ln \phi) .
$$

The appropriate expression for $b_{2}$ follows immediately from relation (59) and the identity $\Delta Q_{r x}=2(\ln \phi)_{r x}, r>1$

$$
b_{2}=\frac{5}{2} Q_{2 x}+c_{2}, \quad c_{2}=\text { arbitrary constant }
$$

the subsequent $b_{i>2}$ being similarly determined (up to arbitrary constants $c_{i}$ ) by using relation (60-62) together with equation (50) and its differential consequences. Setting $c_{4}=c_{5}=0$ and keeping $c_{3}$ as an arbitrary constant, the resulting $L_{5, \text { cov }}(Q)$ can be expressed as follows: 


$$
L_{5, \operatorname{cov}}(Q)=\bar{L}_{5, \operatorname{cov}}(Q)+c_{1} \mathcal{L}_{2}^{2}(Q)+c_{2} L_{3, \operatorname{cov}}(Q)+c_{3} \mathcal{L}_{2}(Q)
$$

with

$$
\bar{L}_{5, \operatorname{cov}}(Q)=\partial_{x}^{5}+\frac{5}{2} Q_{2 x} \partial_{x}^{3}+\frac{15}{4} Q_{3 x} \partial_{x}^{2}+\left(\frac{25}{8} Q_{4 x}+\frac{15}{8} Q_{2 x}^{2}\right) \partial_{x}+\frac{15}{16}\left(Q_{5 x}+2 Q_{2 x} Q_{3 x}\right) .
$$

Again it is easy to verify that the Darboux covariant system in which equation (43) is accompanied by the evolution equation

$$
\phi_{t}+\bar{L}_{5, \operatorname{cov}}(Q) \phi=0
$$

is equivalent to the system (35) and that

$$
\bar{L}_{5, \operatorname{cov}}(Q)=\frac{1}{16} \mathcal{L}_{5}+\frac{15}{16}\left[\partial_{x}^{3}+\left(Q_{2 x}+\lambda\right) \partial_{x}+Q_{3 x}\right]\left[\mathcal{L}_{2}(Q)-\lambda\right]
$$

corresponds precisely to Lax's fifth order generator of isospectral deformations of $\mathcal{L}_{2}(Q)$.

We notice that the appearance of the third order Darboux covariant operator $L_{3 \text {, cov }}(Q)$ as part of $L_{5, \text { cov }}(Q)$ can be regarded as a direct indication of the close relationship between $\mathrm{KdV}$ and $\mathrm{KdV}_{5}$ as members of the same hierarchy.

We also notice that $\mathcal{L}_{2}^{2}(Q)$ represents the actual fourth order operator $L_{4, \text { cov }}(Q)$ to be associated with $\mathcal{L}_{2}(Q)$ for Darboux covariance, hinting, in an elementary way, at the absence of even order flows within the KdV hierarchy.

5. Darboux covariant evolutions and KP. In contrast to the original Lax procedure, the above technique of generating soliton equations through the construction of Darboux covariant linear systems is not restricted to systems associated with a second order eigenvalue equation (43). It is as easy to construct a Darboux covariant system which involves a third order eigenvalue problem and a second order $t$ evolution (interchange of the role of $\mathcal{L}_{2}$ and $L_{3}$ )

$$
\begin{aligned}
& {\left[\partial_{t}+\mathcal{L}_{2}(Q)\right] \phi=0, \quad \mathcal{L}_{2}(Q)=\partial_{x}^{2}+Q_{2 x},} \\
& L_{3}(Q) \phi \equiv \partial_{x}^{3}+b_{2}(Q) \partial_{x}+b_{3}(Q)=\lambda \phi
\end{aligned}
$$

A gauge transformation $G_{\phi}$ generated by a (non vanishing) solution $\phi$ of the system $(69,70)$ maps the operators $\partial_{t}+\mathcal{L}_{2}(Q)$ and $L_{3}(Q)$ onto the similar

$$
\begin{aligned}
& G_{\phi}\left[\partial_{t}+\mathcal{L}_{2}(Q)\right] G_{\phi}^{-1}=\partial_{t}+\mathcal{L}_{2}(\widetilde{Q}=Q+2 \ln \phi), \\
& G_{\phi} L_{3}(Q) G_{\phi}^{-1}=\widetilde{L}_{3}(Q) \equiv \partial_{x}^{3}+\widetilde{b}_{2}(Q) \partial_{x}+\widetilde{b}_{3}(Q),
\end{aligned}
$$

the differences $\Delta b_{i} \equiv \tilde{b}_{i}-b_{i}$ being still given by relation (41). This follows again from differential consequences of equations $(69,70)$, and, in particular from the relation 


$$
\sigma_{t}+\left(\sigma_{x}+\sigma^{2}\right)_{x}+Q_{3 x}=0 .
$$

On account of this relation, and the expressions (41) for $\Delta b_{2}$ and $\Delta b_{3}$, it is clear that the covariance conditions

$$
\widetilde{b}_{i}(Q) \equiv b_{i}(Q)+\Delta b_{i}=b_{i}(\widetilde{Q}=Q+2 \ln \phi), \quad i=2,3,
$$

determine $b_{2}(Q)$ and $b_{3}(Q)$ up to arbitrary constants

$$
b_{2}=\frac{3}{2} Q_{2 x}+c_{2} \quad \text { and } \quad b_{3}=\frac{3}{4}\left(Q_{3 x}-Q_{x t}\right)+c_{3} .
$$

Setting $c_{2}=c_{3}=0$ it is found that $\partial_{t}+\mathcal{L}_{2}(Q)$ and

$$
\widehat{L}_{3, \operatorname{cov}}(Q)=\partial_{x}^{3}+\frac{3}{2} Q_{2 x} \partial_{x}+\frac{3}{4}\left(Q_{3 x}-Q_{x t}\right)
$$

constitute a Darboux covariant Lax pair for a potential version of the Boussinesq equation (23) (with a re-scaled $t$-variable) as

$$
\left[\partial_{t}+\mathcal{L}_{2}(Q), \widehat{L}_{3, \operatorname{cov}}(Q)\right]=-\left(3 Q_{2 t}+Q_{4 x}+3 Q_{2 x}^{2}\right)_{x}
$$

The closely related Darboux covariant Lax systems $(43,54)$ and $(69,70)$ hint at the common origin of the $1+1$ dimensional $\mathrm{KdV}$ and Boussinesq equation as reductions of the $1+2$ dimensional Kadomtsev Petviashvili equation [11]. In fact, it is natural to complete the above analysis by starting from the second order evolution equation (69) and by looking for an associated Darboux covariant $t_{3}$-evolution

$$
\left[\partial_{t_{3}}+L_{3}(Q)\right] \phi=0, \quad L_{3}(Q)=\partial_{x}^{3}+b_{2}(Q) \partial_{x}+b_{3}(Q) .
$$

The appropriate expressions for $b_{2}(Q)$ and $b_{3}(Q)$, are still determined by the conditions (74). In view of relation (41) and equation (73) it is clear that these expressions are given by equation (75) in which $t$ has been replaced by $t_{2}$.

The compatibility condition for the resulting covariant $\left(t_{2}, t_{3}\right)$-system (re-scaling $t_{3}$ )

$$
\begin{aligned}
& {\left[\partial_{t_{2}}+\mathcal{L}_{2}(Q)\right] \phi=0,} \\
& {\left[\partial_{t_{3}}+\mathcal{L}_{3, \text { cov }}(Q)\right] \phi=0, \quad \mathcal{L}_{3, \text { cov }}(Q)=4 \partial_{x}^{3}+6 Q_{2 x} \partial_{x}+3\left(Q_{3 x}-Q_{x t_{2}}\right),}
\end{aligned}
$$

is subject to a condition which can be expressed as the $x$-derivative of an equation which involves a linear combination of $P$-polynomials of weight 4

$$
\left[\partial_{t_{3}}+\mathcal{L}_{3, \operatorname{cov}}(Q), \partial_{t_{2}}+\mathcal{L}_{2}(Q)\right]=\left[P_{x, t_{3}}(Q)+3 P_{2 t_{2}}(Q)+P_{4 x}(Q)\right]_{x}=0,
$$

and which does therefore correspond to a quadratic Hirota expression of degree 4

$$
\mathcal{F}\left(D_{x}, D_{t_{2}}, D_{t_{3}}\right)=D_{x} D_{t_{3}}+3 D_{t_{2}}^{2}+D_{x}^{4}
$$

Equation (81) can be regarded as a potential version of the Kadomtsev-Petviashvili equation 


$$
\left(u_{t_{3}}+u_{3 x}+6 u u_{x}\right)_{x}+3 u_{2 t_{2}}=0
$$

obtained by setting $u=Q_{2 x}$ and by integrating once with respect to $x$.

The present results suggest that a construction of higher order $t_{p}$-evolutions, $p=4,5, \ldots$, in terms of higher operators $\mathcal{L}_{p, \text { cov }}(Q)=\partial_{x}^{p}+b_{2}(Q) \partial_{x}^{p-2}+\cdots+b_{p}(Q)$ which are Darboux covariant with respect to $\mathcal{L}_{2}(Q)$, could be undertaken step by step, so as to produce higher order members of the KP hierarchy. This could be checked by identifying Jimbo and Miwa's higher degree KP equations [11] (involving higher degree Hirota forms) with potential commutator compatibility conditions between two Darboux covariant linear evolutions. In order to do so one must verify that all commutators $\left[\mathcal{L}_{p, \text { cov }}, \mathcal{L}_{2}(Q)\right]$ can be identified (up to differentiation with repect to $x$ ) with expressions involving linear combinations of multidimensional $P$-polynomials $P_{m_{1} t_{1}, \cdots m_{p} t_{p}, \ldots}(Q)$ with $t_{1}=x$.

ACKNOWLEDGements. The authors wish to acknowledge the financial support extended by NATO (expert visit 975655) and that of the "Interuniversity Poles of Attraction Programme, contract nr. P4/08 - Belgian State, Prime Minister's Office Federal Office for Scientific, Technical and Cultural Affairs".

\section{REFERENCES.}

1. R. Hirota, A new form of Bäcklund transformations and its relation to the inverse scattering problem, Progr. Theoret. Phys. 52 (1974), 1498-1512.

2. J. Satsuma, Higher conservation laws for the Korteweg-de Vries equation through Bäcklund transformation, Progr. Theoret. Phys. 52 (1974), 1396-1397.

3. F. Lambert and J. Springael, Construction of Bäcklund transformations with binary Bell polynomials, J. Phys. Soc. Japan 66 (1997), 2211-2213.

4. F. Lambert and J. Springael, On a direct procedure for the disclosure of Lax pairs and Bäcklund transformations, in Chaos, Solitons and Fractals, to appear.

5. C. Gilson, F. Lambert, J. Nimmo and R. Willox, On the combinatorics of the Hirota D-operators, Proc. Roy. Soc. Lond. A 431 (1996), 361-369.

6. V. B. Matveev and M. A. Salle, Darboux transformations and solitons (Springer-Verlag, 1991).

7. E. T. Bell, Exponential polynomials, Ann. of Math. 35 (1934), 258-277.

8. J. J. C. Nimmo and N. C. Freeman, A method of obtaining the $N$-soliton solution of the Boussinesq equation in terms of a Wronskian, Phys. Lett. A 95 (1983), 4-6.

9. W. Oevel and W. Schief, Darboux theorems and the KP hierarchy, in Applications of analytic and geometric methods to nonlinear differential equations (Kluwer Acad. Publ., Dordrecht, 1993).

10. P. D. Lax, Integrals of nonlinear equations of evolution and solitary waves, Comm. Pure Appl. Math. 21 (1968), 467-490.

11. M. Jimbo and T. Miwa, Solitons and infinite dimensional Lie algebras, Publ. Res. Inst. Math. Sci. Kyoto Univ. 19 (1983), 943-1001. 